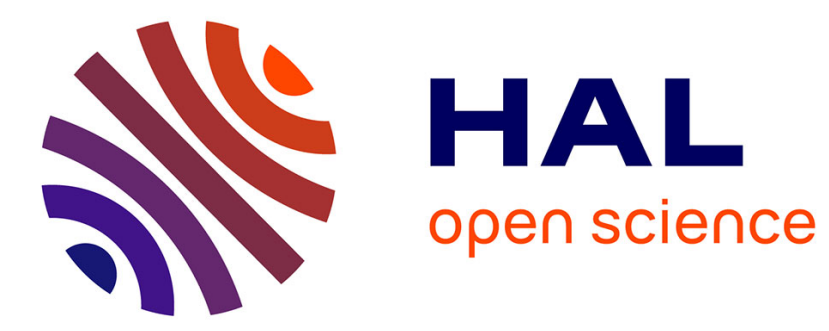

\title{
L'histoire du climat par ses extrêmes. Sources géohistoriques et inondations dans les Pyrénées depuis le Petit Age Glaciaire
}

Jean-Marc Antoine

\section{- To cite this version:}

Jean-Marc Antoine. L'histoire du climat par ses extrêmes. Sources géohistoriques et inondations dans les Pyrénées depuis le Petit Age Glaciaire. Archéologie du Midi Médiéval, 2009, 27, pp. 143-155. halshs-00756650

\section{HAL Id: halshs-00756650 \\ https://shs.hal.science/halshs-00756650}

Submitted on 23 Nov 2012

HAL is a multi-disciplinary open access archive for the deposit and dissemination of scientific research documents, whether they are published or not. The documents may come from teaching and research institutions in France or abroad, or from public or private research centers.
L'archive ouverte pluridisciplinaire HAL, est destinée au dépôt et à la diffusion de documents scientifiques de niveau recherche, publiés ou non, émanant des établissements d'enseignement et de recherche français ou étrangers, des laboratoires publics ou privés. 
Archéologie du Midi Médiéval, t. 27, 2009, pp. 143-155

\title{
L'histoire du climat par ses extrêmes.
}

\section{Sources géohistoriques et inondations dans les Pyrénées depuis le Petit Age Glaciaire.}

\author{
Jean-Marc ANTOINE*
}

RÉSUMÉ. On tente dans cet article de faire le point sur la pertinence à utiliser les événements hydrométéorologiques et géomorphologiques extrêmes, et plus particulièrement les inondations et les crues torrentielles, comme marqueurs des dynamiques climatiques. Après un état des lieux scientifique de cette problématique, est proposé un panorama des sources historiques les plus intéressantes pour retracer l'histoire des inondations. On montre ensuite, à partir de l'exemple pyrénéen, que la dynamique des inondations peut avoir une signification climatique, en particulier dans le contexte du Petit Age Glaciaire. Enfin, sont également évoqués les problèmes méthodologiques qui restent en suspens.

ABSTRACT.

MOTS-CLÉS : histoire des inondations, crises torrentielles, histoire du climat, Petit Age Glaciaire, Pyrénées, Sud-Ouest. KEYWORDS: floods history, torrentials crisis, climate history, Little Ice Age, Pyrenees Mountains, South West France.

\footnotetext{
* Enseignant-chercheur, laboratoire GEODE UMR 5602-CNRS et Département de Géographie de l'Université de Toulouse-Le Mirail, 5 allées Antonio Machado, 31058 Toulouse Cédex 9.
} 
Archéologie du Midi Médiéval, t. 27, 2009, pp. 143-155

Aborder l'histoire du climat par ses extrêmes n'est pas anodin et sans présenter quelques difficultés. Pour l'homme de la rue, et depuis des temps immémoriaux, lier les catastrophes hydrométéorologiques et le climat va de soi, les premières passant invariablement pour être la preuve bien tangible des « dérèglements » du second. Et ce d'autant que les « experts » s'accordent aujourd'hui pour associer étroitement changements climatiques et recrudescence des phénomènes météorologiques et hydrométéorologiques extrêmes (Houghton et al., 2001 ; Pachauri et al. 2007). Toutes choses égales, ce qui vaut pour le présent pouvant valoir également pour le passé, la dynamique historique des inondations catastrophiques pourrait donc aussi rendre compte d'évolutions climatiques anciennes, au premier rang desquelles celle du Petit Age Glaciaire.

Etablir cette relation n'est pas cependant chose aisée, en raison tout d'abord du conflit d'échelle spatio-temporelle entre inondation et climat, c'est-à-dire entre un événement ponctuel dans l'espace et dans le temps et une tendance moyenne dans les mêmes termes. D'un autre côté, et au-delà du jeu des processus écologiques, les catastrophes hydrométéorologiques sont aussi le produit de processus sociaux et sociétaux divers, jouant aussi bien la dynamique objective des phénomènes que sur la perception que l'on peut en avoir a posteriori (Bravard, 2006; Antoine, 2007, à paraître) La question est donc de savoir dans quelle mesure les événements hydro-météorologiques extrêmes, et plus précisément les inondations, peuvent passer pour un marqueur des évolutions climatiques, et notamment du Petit Age Glaciaire.

\section{ETAT DES LIEUX DE LA PROBLEMATIQUE}

D'emblée, on se doit de constater que les historiens, spécialistes a priori tout désignés d'une histoire des catastrophes naturelles, n'ont jamais manifesté un grand intérêt pour la question, notamment en France. Quand ils s'y sont risqués, c'est soit de façon occasionnelle (Berlioz, 1987 et 1993 ; Desplat, 1988, 1994 ; Bennassar, 1996 ), soit en marge d'études débordant cette thématique, et relevant la plupart du temps et plus largement de l'histoire des mentalités (Delumeau et al., 1991 ; Briffaud, 1993, 1994), de l'histoire de l'environnement (Walter, 1990 ; Beck et al., 1993 ; Pichard, 1999; Delort et al., 2001) ou de l'histoire du climat (Le Roy Ladurie, 1967 ; Alexandre, 1987). Il est d'ailleurs symptomatique que le dossier consacré par les Annales ESC à « Histoire et environnement» en 1974 ne compte qu'une seule contribution d'historien, et que les divers programmes du CNRS sur l'environnement, voire même sur l'histoire de l'environnement, engagés dès le milieu des années 1980 (PIREN, PIREVS, PEVS...), n'aient que très peu attiré les historiens « de métier».

C'est hors de France qu'il faut aller chercher les seuls véritables historiens spécialistes de la question, en Espagne par exemple (Barriendos, 1993), mais surtout en Suisse, où depuis plus de vingt ans Ch. Pfister (Pfister, 1988) travaille sur la problématique des catastrophes naturelles, qu'il lie en outre à celle des changements climatiques. Plus récemment et plus ponctuellement, un autre historien suisse, F. Walter (Walter et al., 2006 ; Walter, 2008), s'est aussi intéressé à cette question, mais plutôt dans ses dimensions culturelles, de même que l'historien autrichien Ch. Rohr (2005).

C'est aussi depuis peu que les historiens français ont été amené à s'intéresser à l'histoire des inondations: G. Pichard (Pichard, 1995, 2004) dans le cadre et à la suite de sa thèse sur l'environnement rural en Provence, P. Allard (Allard, 2000 ; Picon et al., 2006) depuis son HDR sur le risque d'inondation en Camargue, E. Garnier (Garnier, 2006a, 2006b) dans le contexte plus général de ses travaux sur l'histoire de l'environnement, et surtout les chercheurs grenoblois du LARHRA (Granet-Abisset et al., 2001; Favier, 2002; Favier et al., 2005), seule équipe d'historiens à avoir réfléchi à l'histoire des risques et des catastrophes naturelles, dans la foulée de la thèse et des travaux de D. Cœur (Cœur et al., 2000 ; Cœur, 2003). 
Curieusement, et G. Massard-Guilbaud (2002) l'a bien noté, les historiens ont été précédés par les géographes qui, a contrario, ont été plus nombreux à s'emparer de la question et beaucoup plus précocement, tant à l'étranger (Gottschalk, 1971-1977 ; Lamb, 1991 ; Pavese et al., 1995 ; Benito et al., 1996; Brazdil, 1998, Glaser et al., 2003, 2004), qu'en France (Métailié, 1987, 1991 ; Desailly, 1990 ; Antoine, 1992, 1993 ; Antoine et al., 1993, 1995 ; Meschinet de Richemont, 1997). Il n'y a donc pas eu véritablement de mise au point méthodologique sur les sources historiques utilisables pour une histoire événementielle des catastrophes naturelles, si ce n'est marginalement à l'occasion des ouvrages relatifs aux sources de l'histoire de l'environnement publiés sous la direction de A. Corvol (1995, 1999, 2003), ou de façon confidentielle par nousmêmes (Antoine et al., 2000, 2001). Pas plus qu'il n'y a eu de réflexion méthodologique venant éclaircir les relations pouvant être établies entre dynamique des inondations et climat.

\section{SOURCES ET DONNEES DISPONIBLES POUR UNE HISTOIRE DES INONDATIONS}

Les sources privilégiées pour reconstituer l'histoire récente des inondations - i.e. depuis le début du $\mathrm{XX}^{\mathrm{e}}$ siècle -, sont d'origine administrative, et donc consultables principalement auprès des services intéressés à la gestion de l'eau et au risque d'inondation (DDE, DIREN, DDAF, services RTM de l'ONF...). De plus en plus souvent aujourd'hui, les informations relatives aux inondations y sont d'ailleurs conservées et formalisées dans des bases de données informatisées (Fichier CatNat, base de données GASPAR et Banque Hydro du Ministère de l'Ecologie et du Développement Durable, Bases de données Evénements des services RTM ...). Dans la perspective d'une histoire des inondations et du climat, les données archivées dans ces bases couvrent donc au mieux la période de sortie du Petit Age Glaciaire.

Pour les périodes antérieures au $\mathrm{XX}^{\mathrm{e}}$ siècle, voire aux années 1940, les sources sur les inondations et crues torrentielles sont de nature archivistique, et sont donc conservées dans les différents services d'archives publiques: Archives Nationales, Archives Municipales et Archives Départementales. Très souvent cependant, la seule consultation de ces dernières suffit à récoler l'information : les véritables services d'archives municipales sont rares, la plupart des communes versant leurs fonds aux services départementaux, alors que les documents des Archives Nationales doublonnent souvent avec ceux conservés à l'échelon départemental.

Quels que soient les services d'archives, tous font la distinction entre les documents antérieurs à la Révolution et ceux postérieurs (Fig. 1). Cette coupure politique est aussi qualitative : avec la centralisation de l'administration et l'organisation hiérarchisée des différents ministères et de leur relais départementaux, les archives postérieures à 1789 sont bien plus riches et bien mieux classées que celles de l'Ancien Régime.

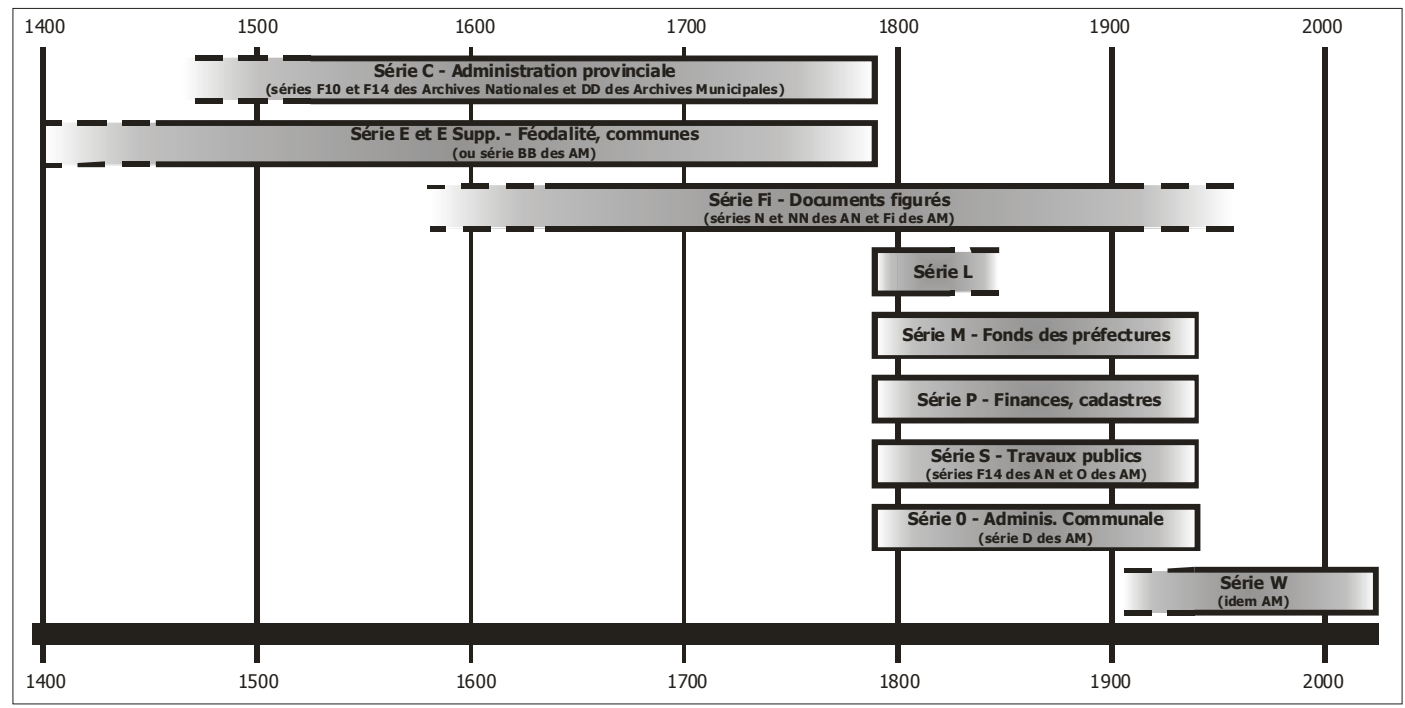


Fig. 1 - Les séries pertinentes des archives départementales et leur couverture temporelle

Pour les fonds d'Ancien Régime, la série phare des Archives Départementales est la série $C$ ou, à défaut, les sous-séries $F^{10}$ et $F^{14}$ des Archives Nationales et la série $D D$ des Archives Municipales. Abondante et intéressante surtout à partir des $\mathrm{XVI}^{\mathrm{e}}-\mathrm{XVII}$ siècles, elle rassemble tous les documents relatifs à l'Administration provinciale. Les plus productifs à propos des inondations sont ceux relatifs :

- aux impositions: demandes d'exemption ou de dégrèvement d'impôt émanant des communautés ou des particuliers, procès-verbaux de vérification des dommages et de répartition des secours...

- aux ponts et chaussées et aux travaux publics : entretien et réparation des routes et ponts, moulins, petite hydraulique, endiguements, voies navigables...

- à la correspondance des intendants avec le contrôleur général ;

- aux affaires communales : requêtes, délibérations des communes, travaux publics...

La série $E$ ou $E$ Supplément «Féodalité, communes, bourgeoisie, familles » (et son équivalent communal, la série $B B$ ) peut également contenir des informations intéressant les inondations. Les sous-séries les plus riches sont celles concernant les sinistres, l'agriculture, les travaux publics, les délibérations communales, l'Etat-Civil et les registres paroissiaux. Cependant, chaque commune du département disposant d'un fonds propre aux Archives Départementales, le dépouillement de la série $E$ peut être long et fastidieux. Et d'autant plus si l'on s'essaye à l'examen des fonds notariaux conservés également dans cette série, et dans l'immense masse desquels sont parfois consignées des informations sur les inondations nulle part ailleurs répertoriées.

On doit enfin souligner que toutes les séries d'Ancien Régime indistinctement peuvent contenir des «affiches », «placards » et autres «canards", sorte de presse écrite avant la lettre. Bon nombre d'entre eux sont relatifs aux «malheurs des temps», parmi lesquels les inondations occupent une place de choix.

La série $F i$, «Documents figurés », est apériodique et ne connaît pas la coupure de 1789. C'est aussi sans doute la série la plus «géographique » : cartes, plans, gravures et lithographies, cartes postales anciennes et photographies rendent compte parfois, et a priori de façon «objective », des inondations catastrophiques replacées dans leurs contextes spatiaux, environnementaux et paysagers (Photo. 1).

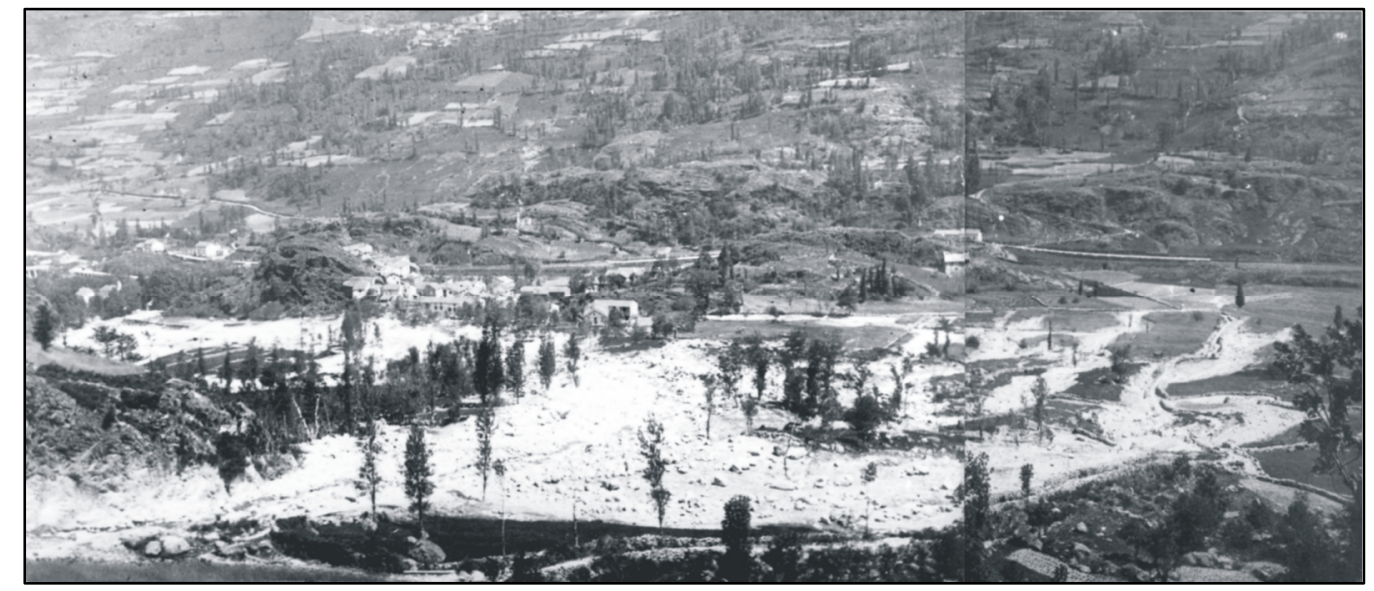

Photo. 1 - Les laves torrentielles du 23 mai 1910 à Perles-et-Castelet, Ariège 
Passée la période révolutionnaire, couverte par une série spécifique, la série $L$, quatre séries peuvent s'avérer intéressantes parmi les séries modernes des Archives Départementales, dont les documents peuvent aller jusqu'aux années 1940 :

- la série $M$ « Fonds des préfectures » ou «Administration des départements » : on y trouve parfois des dossiers détaillés sur les «Sinistres et calamités », toujours des états de pertes et des rapports de gendarmerie établis après les grandes inondations ;

- la série $S$ «Travaux publics» (et son équivalent des Archives Municipales, la série $O$ ), constituée pour l'essentiel du fonds «Ponts et Chaussées», et dans laquelle sont conservées les archives des services hydrauliques et des services d'annonce des crues, avec rapports, plans, côtes de crues, parfois des photographies sur les grandes inondations ; son équivalent «Eaux et Forêts » se trouve dans la série $P$ «Finances, cadastres », laquelle accueille également, dans les départements montagnards, le fonds RTM.

- enfin la série $O$ (et sa jumelle des Archives Municipales, la série $D$ ), consacrée à l'administration communale, pendant moderne de la série $E$ ou E Supplément ; structurée en sous-séries similaires, les plus prometteuses sont celles dédiées aux « Travaux publics », « Sinistres et calamités », « Agriculture »...

Les Archives Départementales accueillent enfin la série $W$, alimentée par les versements postérieurs à 1940 émanant ordinairement des administrations, notamment celles des « Ponte \& Chaussées » et des «Eaux \& Forêts ». Cette série est cependant d'un abord difficile, les documents étant archivés au fur et à mesure de leur versement, généralement sans aucun classement.

Sur un autre registre, qualité, précision et exhaustivité des sources sont très hétérogènes d'une série à l'autre, d'un service d'archives à un autre. La façon dont sont recensés les événements varie par exemple de la simple mention de survenance dans une commune (dans des états de pertes par exemple) à leur localisation précise et leur description détaillée, quantifiée en termes de dégâts matériels mais aussi de pluviométrie, de hauteur d'eau, de vitesse de submersion, de volumes des débits solides transportés ou déposés, et illustrée par des plans de champs d'inondation ou des photographies des lieux inondés. D'une manière générale, mais pas systématique, la précision gagne avec le temps, les événements les plus récents étant d'ordinaire les mieux renseignés.

A côté de ces sources de première main, les sources imprimées ne doivent pas être négligées (Fig. 2). Elles peuvent être d'une aide fort précieuse pour identifier dans un service d'archives, les séries, les fonds ou les liasses a priori les plus prometteurs. On pense par exemple aux Annales communales, tenues parfois depuis les $\mathrm{XV}^{\mathrm{e}}-\mathrm{XVI}^{\mathrm{e}}$ siècles ${ }^{(1)}$, ou aux synthèses chronologiques et événementielles sur les inondations publiées aux $\mathrm{XIX}^{\mathrm{e}}-\mathrm{XX}^{\mathrm{e}}$ siècles ${ }^{(2)}$, dans la lignée de la somme de M. Champion éditée à partir de $1862^{(3)}$. Ces compilations sont néanmoins souvent lacunaires et leurs informations parfois invérifiables. Les erreurs de transcription des documents originaux ou de recopie des sources de seconde main ne sont pas rares, et les recoupements souvent rendus difficiles lorsque les sources originales sont privées, ont disparue ou ont été reclassées.

Enfin, la presse, bien qu'elle pose les problèmes les plus aigus de critique des sources et des données, n'en demeure pas moins d'un secours non négligeable, notamment pour les événements survenus au $\mathrm{XIX}^{\mathrm{e}}$ siècle et au début du $\mathrm{XX}^{\mathrm{e}}$ siècle, à l'âge d'or de la presse locale et régionale. Les Archives Départementales du Gers conservent par exemple quelques 350 quotidiens,

\footnotetext{
1 - Par exemple à Toulouse : G. De Lafaille (G.), 1687-1701, Annales de la Ville de Toulouse, Toulouse, Coulomyes, 2 tomes.

2 - On peut citer parmi de nombreuses références, Guiraud de Saint-Marsal (R.), « Mémoire sur les inondations occasionnées par les crues de la Têt et de la Basse aux environs de Perpignan », Bull. Soc. Agric. Sc. Lit. des Pyrénées-Orientales, tome X, 1856, ou encore Gaussen (I.), Le Vidourle et ses vidourlades, Nîmes, Editions Méridionales, 1937, 200 p..

3 - Champion (M.), Les inondations en France du VI siècle à nos jours, Paris, Dunod, 1862-1868, 6 vol..
} 
hebdomadaires, mensuels couvrant ensemble la période allant de 1814 à aujourd'hui (Antoine $e t$ $a l ., 2000)$.

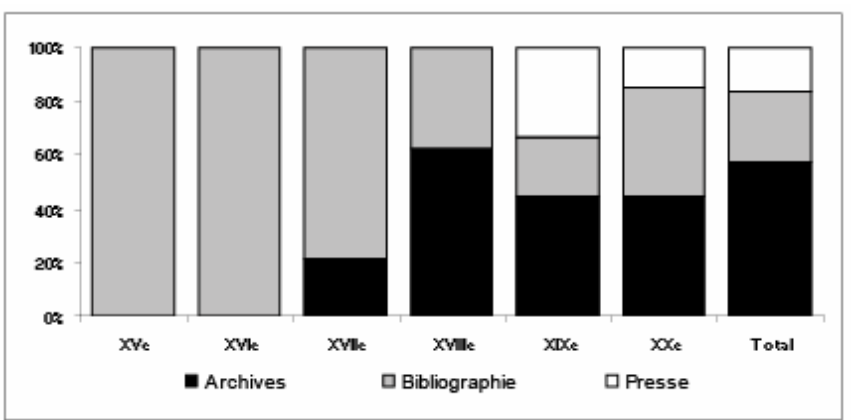

Fig. 2 - L'origine des informations sur les inondations historiques dans le Gers ( $X^{\mathrm{e}}-\mathrm{XX}^{\mathrm{e}}$ siècles) (D'après Antoine et al., 2000)

$\mathrm{Au}$ final, les sources fondamentales pour l'histoire des inondations jusqu'au milieu du XIX ${ }^{\mathrm{e}}$ siècle sont les sources archivistiques, dont la richesse dans l'absolu a pour revers un temps de consultation très long. Il peut être significativement réduit par le dépouillement de sources de seconde main (sources imprimées, presse...), qui doivent cependant être maniées avec précautions tant elles ne peuvent pas toujours être vérifiées et recoupées.

\section{LES ENSEIGNEMENTS DE LA DYNAMIQUE HISTORIQUE DES INONDATIONS ET DES CRUES TORRENTIELLES DANS LES PYRENEES}

Nos travaux au sein du pôle "Risques et catastrophes naturelles » du laboratoire GEODE UMR-5602 CNRS, portent sur l'histoire des catastrophes naturelles dans les Pyrénées et sur leur piémont, inondations et crues torrentielles essentiellement (Antoine, 1992; Antoine et al., 1993, 1995, 2000, 2007 ; Brazdil et al., 1999). La chronologie établie depuis une quinzaine d'années permet d'avoir une vision assez claire de la dynamique des phénomènes depuis le XVII ${ }^{\mathrm{e}}$ siècle, mais reste plus lacunaire pour les périodes antérieures. Nous disposons donc d'éléments qui couvrent la fin du Petit Age Glaciaire et la sortie de cette période.

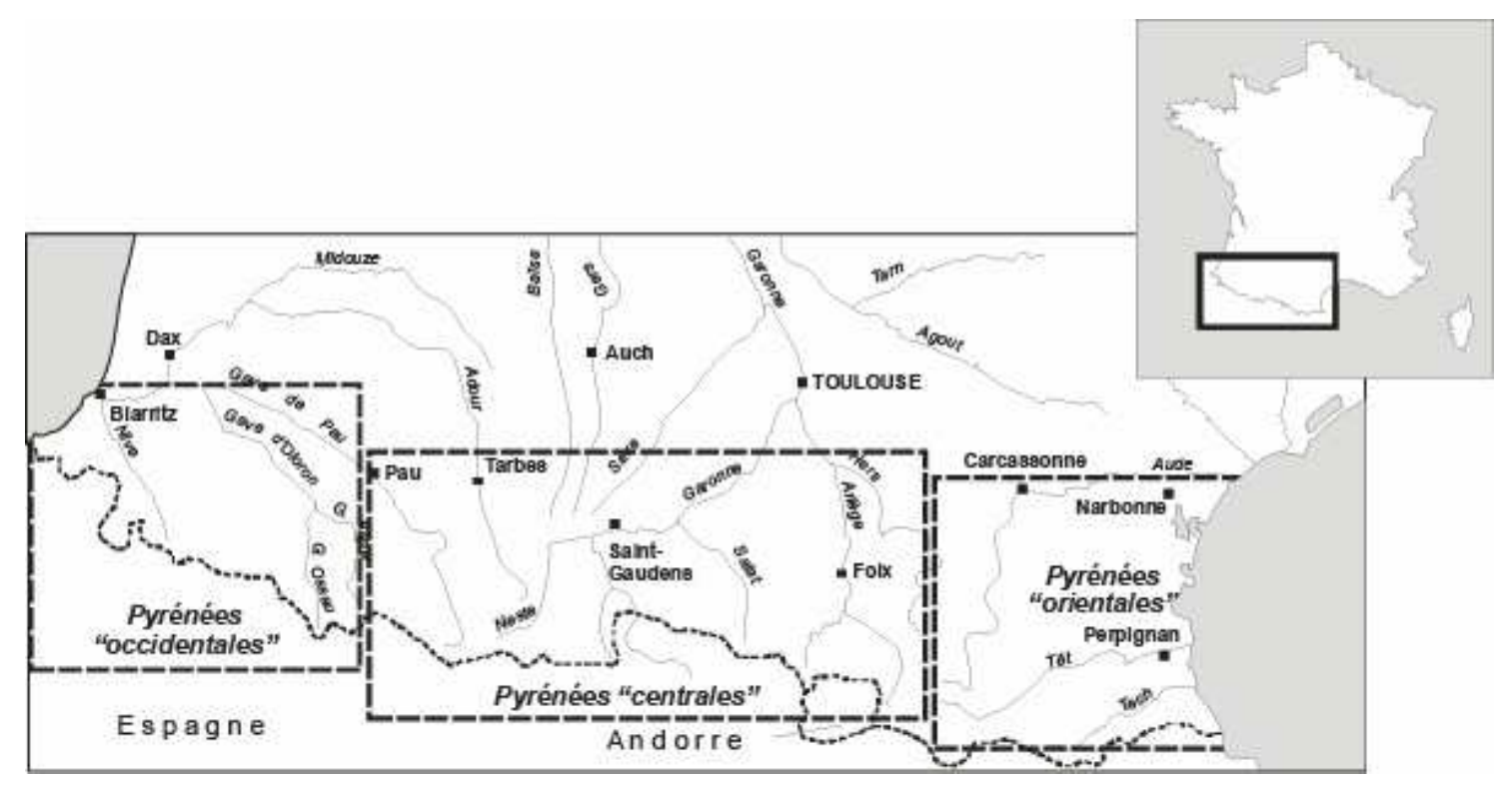

Fig. 3 - Carte de localisation 
Les résultats exposés ici s'appuient d'abord sur une mise en forme et une synthèse des chronologies détaillées d'événements plus ou moins catastrophiques, qui ont été établies au sein du laboratoire GEODE pour diverses vallées de la chaîne (Fig. 3), à l'occasion :

- de travaux universitaires : thèses, mémoires de Master1 et 2 ;

- de programmes de recherche: PIREN-CNRS «Histoire de l'environnement» (19881991) ou Programme SRETIE (1990-1993) ${ }^{(4)}$;

- de la conception/réalisation/alimentation pour les services de Restauration des Terrains en Montagne (ONF/DDAF) de bases de données départementales sur les risques naturels (départements de l'Ariège, de la Haute-Garonne, des Hautes-Pyrénées et des PyrénéesAtlantiques).

Ce travail est aussi le fruit de recherches archivistiques et bibliographiques complémentaires effectuées sur les secteurs les moins bien couverts par les travaux antérieurs (Aude, Pays Basque et Béarn notamment), dans le cadre du programme ECLIPSE (Environnements et climats du passé) du CNRS en 2001-2004. Il s'agissait là d'analyser les relations entre le Petit Age Glaciaire et la dynamique historique des inondations, crues torrentielles et avalanches survenues dans les vallées pyrénéennes au cours de l'Histoire, et répertoriées par les sources archivistiques et bibliographiques.

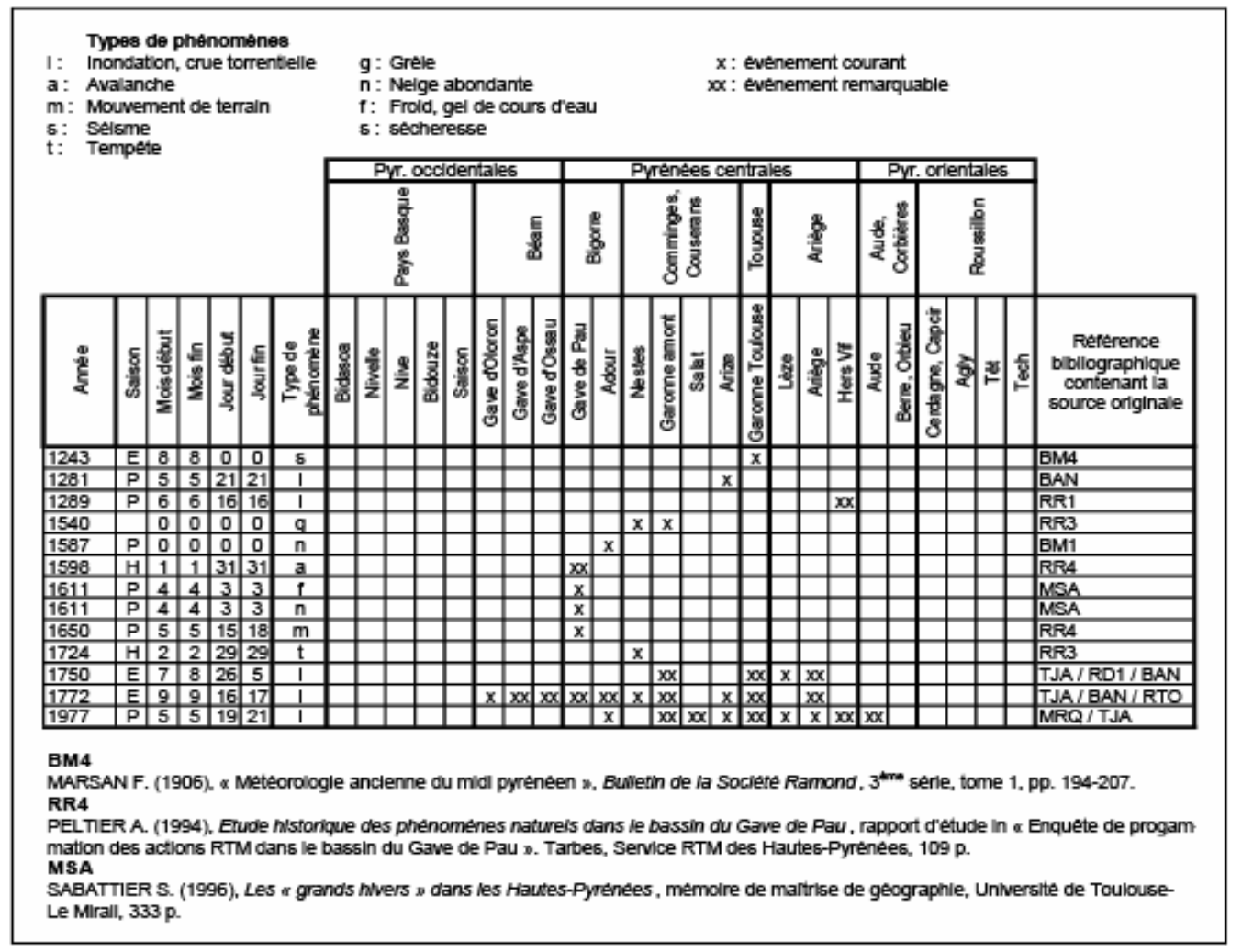

Fig. 4 - Extrait du tableau de recensement des phénomènes naturels vecteurs de risques dans les Pyrénées (1200-2000) (J.M. Antoine, B. Desailly, A. Peltier)

\footnotetext{
4 - A l'occasion aussi de programmes de recherche appliquée auprès des ministères ou d'organismes publics tels le programme "Faisabilité de bases de données historiques sur les inondations - Base nationale et bases départementales », Ministère de l'Environnement et de l'Aménagement du Territoire, CERTU Lyon (2000), ou encore le programme «Cartographie historique des phénomènes naturels à risque dans les Pyrénées », DIREN Midi-Pyrénées, service RTM Pyrénées (2003-2005).
} 
Les données ont été formalisées dans un tableau récapitulatif (Fig. 4) qui recense 2431 événements hydro-climatiques divers survenus entre le IX ${ }^{\mathrm{e}}$ siècle et 2001 : inondations, grêles, tempêtes, avalanches, vagues de froid/gel, mouvements de terrain. Ces événements sont recensés et classés selon trois niveaux spatiaux : bassins hydrographiques, pays pyrénéens puis grands secteurs de la chaîne. Chaque fois que la qualité des sources l'a permis, on a distingué les «événements courants » des «événements remarquables » (c'est-à-dire ceux ayant détruit ou fortement endommagé des bâtiments, ou bien ayant fait des victimes, ou encore ceux sensibles dans au moins deux vallées).

Pour les résultats exposés ici, nous n'avons retenu dans ce corpus que les inondations et crues torrentielles, lesquelles représentent néanmoins plus de 50\% du total, soit 1270 événements survenus entre le IX ${ }^{\mathrm{e}}$ siècle et 2001. L'examen de leur succession historique montre deux types d'évolution.

\subsection{Des évolutions quantitatives}

On notera au préalable que ce n'est qu'à partir des $\mathrm{XVI}^{\mathrm{e}}-\mathrm{XVII}{ }^{\mathrm{e}}$ siècles (soit pour 1241 événements valléens correspondant à 817 événements "pyrénéens ») que l'on peut esquisser une analyse statistique de l'évolution historique des inondations et des crues torrentielles dans les Pyrénées. Avant cette date, l'exhaustivité des informations est en effet très variable selon les vallées. Même pour les vallées les mieux pourvues, l'effectif n'est en outre pas assez abondant pour avoir une signification statistique. Cette restriction ne pose d'ailleurs pas de problème majeur pour notre analyse, puisque la $2^{\text {nde }}$ partie du $\mathrm{XVI}^{\mathrm{e}}$ siècle passe pour inaugurer la période la plus froide du Petit Age Glaciaire, qui se termine ensuite vers 1850.

Le Petit Age Glaciaire n'apparaît cependant pas indiscutablement en tant que tel dans la chronologie, ou en tout cas, pas de façon homogène (Fig. 5). Il fait peu de doute qu'avant le XVIII siècle, en raison de sources d'information plus rares et moins précises, les phénomènes sont sousreprésentés, alors que, pour des raisons inverses, ils sont certainement sur-représentés au $\mathrm{XX}^{\mathrm{e}}$ siècle. Si on ne peut replacer la période du Petit Age Glaciaire dans un large contexte historique, notamment à propos des modalités d'entrée dans la période, les données dont on dispose permettent néanmoins de mettre en perspective la dynamique interne et les modalités de sortie de la période.
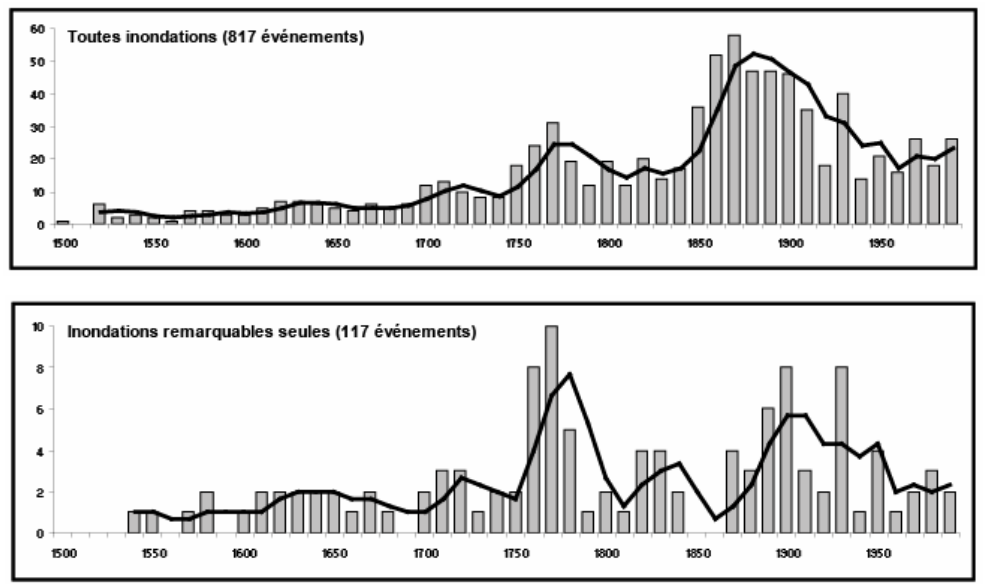

Fig. 5 - Les inondations et crues torrentielles recensées dans les Pyrénées (1500-2000) 
On observe d'abord que la dynamique historique des phénomènes n'est pas linéaire, faisant visiblement alterner périodes d'exacerbation et périodes de moindre activité, s'étalant sur une à quelques décennies. Apparaissent ainsi particulièrement actives les décennies 1750-1780, 18501910 et, dans une moindre mesure, 1570-1590, 1620-1640 et 1700-1720. L'examen des seules inondations remarquables (i.e. particulièrement dommageables ou ayant touché au moins deux vallées) ne remets pas fondamentalement en cause ce constat, malgré quelques distorsions et décalages dans le temps (période de recrudescence de la fin du XIX ${ }^{\mathrm{e}}$ siècle qui commencerait avec la décennie 1850 pour les événements courants et avec la décennie 1870 pour les événements remarquables par exemple). L'évolution des phénomènes reste scandée par des rythmes comparables : on retrouve une certaine aggravation des phénomènes à partir des années 1580 et, surtout, l'individualisation de trois des quatre périodes de recrudescence précédemment évoquées (1710-1720, 1760-1780 et 1870-1910). Par contre, et contrairement à ce que montre la dynamique des événements courants, la péjoration de la fin du XVIII ${ }^{\mathrm{e}}$ siècle semble plus marquée que celle de la seconde partie du $\mathrm{XIX}^{\mathrm{e}}$ concernant les grandes crues. Ceci nous conduit à formuler trois hypothèses non exclusives :

- la fin du XIX $\mathrm{e}^{\mathrm{e}}$ siècle aurait connu une plus grande fréquence des phénomènes et la fin du $\mathrm{XVIII}^{\mathrm{e}}$ de plus grandes intensités ;

- la période de crise du XIX ${ }^{\mathrm{e}}$ siècle relève sans doute de ressorts climatiques mais sans doute aussi d'une plus grande sensibilité des versants montagnards à la torrentialité, doublée d'un recensement plus systématique des phénomènes par la société et donc par les sources ; par conséquent, la fin du XVIII ${ }^{\mathrm{e}}$ siècle pourrait passer pour une période de recrudescence « objective» des phénomènes, au-delà des vicissitudes de leur consignation dans les archives ;

- la fin du XVIII ${ }^{\mathrm{e}}$ serait le siège d'une vraie crise climatique, la fin du XIX ${ }^{\mathrm{e}}$ conjuguant pour sa part une crise climatique et une crise environnementale au sens de J.-P. Bravard (Bravard, 2006).

Il semblerait donc que la dynamique historique des inondations puisse effectivement marquer l'occurrence du Petit Age Glaciaire, non par la matérialisation d'une période monolithique d'exacerbation des dynamiques hydrologique et torrentielle, mais plutôt par la mise en évidence d'une phase multiséculaire très contrastée du point de vue de la fréquence des phénomènes. Ce qui ne va pas à l'encontre de ce que l'on sait du Petit Age Glaciaire, que la plupart des auteurs s'accordent à dépeindre comme une période caractérisée par un climat erratique, extrêmement variable d'une saison à l'autre et d'une année à l'autre, alternant hivers sévères et hivers doux, étés chauds et secs et étés frais et «pourris » (Lamb, 1977, 1995 ; Fagan, 2000).

Enfin, venant en quelque sorte valider les tendances pyrénéennes, on notera par ailleurs que cette évolution rythmée a été observée ailleurs, même si c'est avec des bornes chronologiques parfois discordantes : en Catalogne espagnole (Barriendos Vallvé, 1993 ; Barriendos Vallvé et al., 1998), sur le Rhône (Pichard, 1995 ; Arnaud-Fassetta et al., 1999), la moyenne Durance (Miramont et al., 1997), les cours d'eau italiens (Pavese et al., 1995) ou encore en Europe Centrale (Brazdil et al., 1999).

\subsection{Des évolutions «qualitatives »}

Au-delà des aspects quantitatifs, les aspects qualitatifs de la dynamique historique des inondations pyrénéennes traduisent également la singularité du Petit Age Glaciaire. On entend par aspects qualitatifs, d'une part la plus ou moins grande intensité apparente des phénomènes et, d'autre part, leurs saisons de survenance.

On vient de le voir, certaines époques apparaissent peu touchées par les phénomènes, même si elles peuvent être le siège de grandes inondations : il en est ainsi de la période 1720-1750 où la 
crue du 12 septembre 1727 fait pourtant 54 morts et détruit 448 maisons à Toulouse (Galabert, 1918), ou de la période 1780-1850 au sein de laquelle surviennent pourtant, toujours à Toulouse, deux des plus grandes inondations répertoriées, les 21 mai 1827 ( $4^{\mathrm{e}}$ rang historique) et 30 mai 1835 ( $2^{\mathrm{e}}$ rang) (SMEPAG, 1989). Mais ces événements restent isolés, contrairement à ce que l'on observe lors des périodes de grande activité hydrologique et torrentielle (1750-1780 et 18701910) : celles-ci conjuguent à la fois plus grande fréquence, on l'a vu plus haut, mais aussi plus grande intensité des événements, donnant lieu à de véritables crises hydrologiques et torrentielles. Et si signature hydrologico-torrentielle du Petit Age Glaciaire il y a, c'est précisément dans ces crises qu'il faut la chercher.

Leur caractère et leur déroulement ont déjà été décrits (Métailié, 1987; Desailly, 1990 ; Antoine, 1992 ; Antoine et al., 1993, 1995...) et récemment remis en perspective (Métailié 2006 ; Antoine et al., 2007, à paraître). Elles combinent des événements hydrologico-torrentiels de faible fréquence - généralisés à la chaîne pyrénéenne ou plus localisés -, la permanence d'une activité torrentielle plus courante, et enfin, d'autres phénomènes hydro-climatiques extrêmes (avalanches, glissement de terrain, grands froids et sècheresses...). Ces phénomènes se succèdent sur quelques décennies et se concentrent même parfois sur certaines années particulièrement funestes (Fig. 6). Si on prend l'exemple de la crise de la fin du XVIII siècle, courant des années 1750 aux années 1780, elle est le siège :

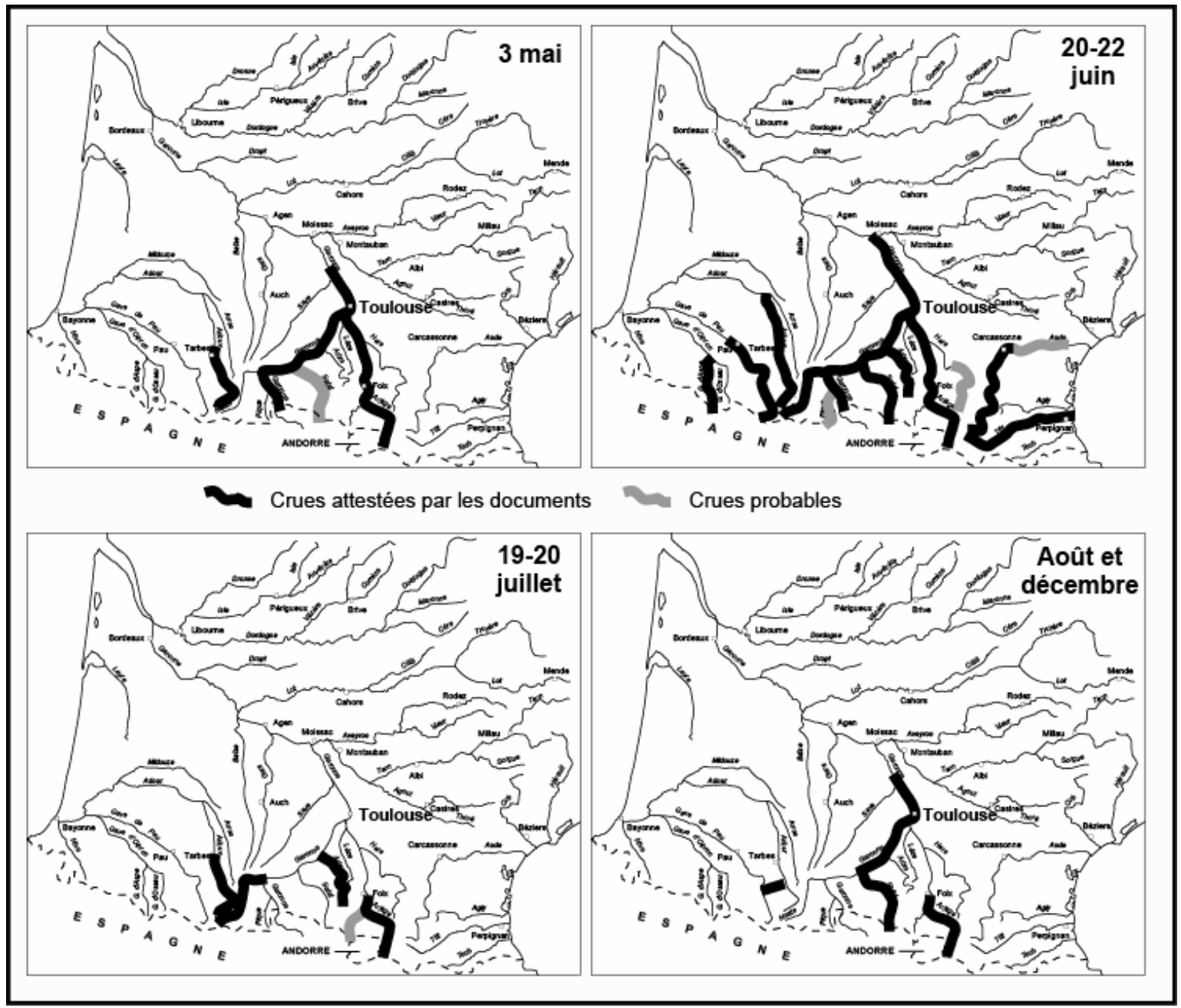

Fig. 6 - Les inondations de l'année 1765 dans les Pyrénées 
- d'inondations catastrophiques généralisées en juin 1765 (du gave d'Aspe à la Têt et sur le piémont), avril 1770 (du gave d'Oloron à l'Ariège et au piémont), septembre 1772 (du Gave d'Oloron à la Têt et au piémont $)^{(5)} \ldots$

- d'inondations catastrophiques localisées; en Haute-Ariège par exemple, en sus des inondations mentionnées ci-dessus (celle de 1772 détruit l'église de Tarascon), on note les catastrophes torrentielles d'août 1750 (14 morts à Miglos; 25 maisons détruites à Luzenac), d'août 1762 (11 morts à Miglos ; maisons détruites à Aulos, Lapège et Bouan), d'août 1774 (8 morts à Prades), de juin 1777 (maisons détruites à Arnave), sans compter de puissants engravements en $1750,1762,1770,1772,1774,1776$ et $1777 \ldots$

- de grands hivers avalancheux, tel celui de 1770 dans toutes les vallées, du Gave d'Aspe à l'Ariège (plusieurs avalanches meurtrières en janvier 1770) ;

- d'années particulièrement funestes, par la succession d'inondations d'un bout à l'autre de la chaîne (mai, juin, juillet, août et décembre 1765, du gave de Pau à la Têt et l'Aude) (Fig. 6), parfois dans les mêmes vallées (3 grandes inondations dans la vallée de l'Ariège en avril et mai 1770), ou encore par l'enchaînement de phénomènes hydrométéorologiques extrêmes (les inondations d'avril et mai 1770 avaient été précédées par de puissantes avalanches en janvier).

Cette période, tout comme la fin du $\mathrm{XIX}^{\mathrm{e}}$ siècle, présente donc bien une singularité de la dynamique hydrologico-torrentielle, et au-delà sans doute climatique, car on comprendrait mal sinon pourquoi, passées les années 1790, fréquence et intensité des phénomènes faiblissent jusqu'aux années 1850, avant de repartir à la hausse.

Un autre indicateur qualitatif de la singularité des inondations et crues torrentielles au cours du Petit Age Glaciaire réside dans les saisons de survenance des phénomènes. Elles connaissent une grande variabilité spatio-temporelle, aussi bien tout au long de la chaîne pour une même période, que pour un même cours d'eau au cours de périodes successives. Or, à chaque saison est associé un scénario hydro-météorologique spécifique donnant un type de crue particulier. La variabilité saisonnière de survenance des crues implique donc nécessairement une variabilité des scénarii hydro-météorologiques, et par conséquent, une possible variabilité des mécanismes climatiques. On a sans doute là un moyen permettant de relier inondations et climat.

L'évolution de la répartition saisonnière des inondations a été examinée en fonction des grands secteurs de la chaîne (aile occidentale, centre, aile orientale), et sur des périodes déterminées $a$ priori, en fonction de ce que l'on sait du Petit Age Glaciaire en Europe de l'Ouest : période du Petit Age Glaciaire s.s. (1550-1849), période de sortie du Petit Age Glaciaire (1850-1949) et période contemporaine (1950-1999).

On observe tout d'abord qu'à notre époque (période 1950-1999), selon la position des vallées le long de la chaîne, la répartition saisonnière des inondations est très différente, conformément aux spécificités des domaines climatiques concernés (Fig. 7) :

- crues d'hiver liées à des circulations zonales d'ouest pour le domaine atlantique ;

- crues d'automne liées à des circulations méridiennes ou bien à des dépressions d'ouest à trajectoire très méridionale pour le domaine méditerranéen ;

- crues de printemps pour le domaine de transition au centre de la chaîne.

5 - Sur bon nombre de cours d'eau du bassin de la Garonne, les inondations de 1770 et 1772 arrivent souvent en $2^{\text {ème }}$ position, généralement après celle de juin 1875, voire sont les plus catastrophiques connues. On notera aussi pour la partie méditerranéenne, ou sous influence méditerranéenne, que la fin du XVIII ${ }^{\mathrm{e}}$ siècle est aussi le siège d'inondations parmi les plus grandes observées : celle d'octobre 1763 en Conflent et Vallespir (Pyrénées-Orientales) se place juste après celle d'octobre 1940, à l'image de celle de novembre 1766 sur le Tarn qui vient juste après celle de mars 1930. 


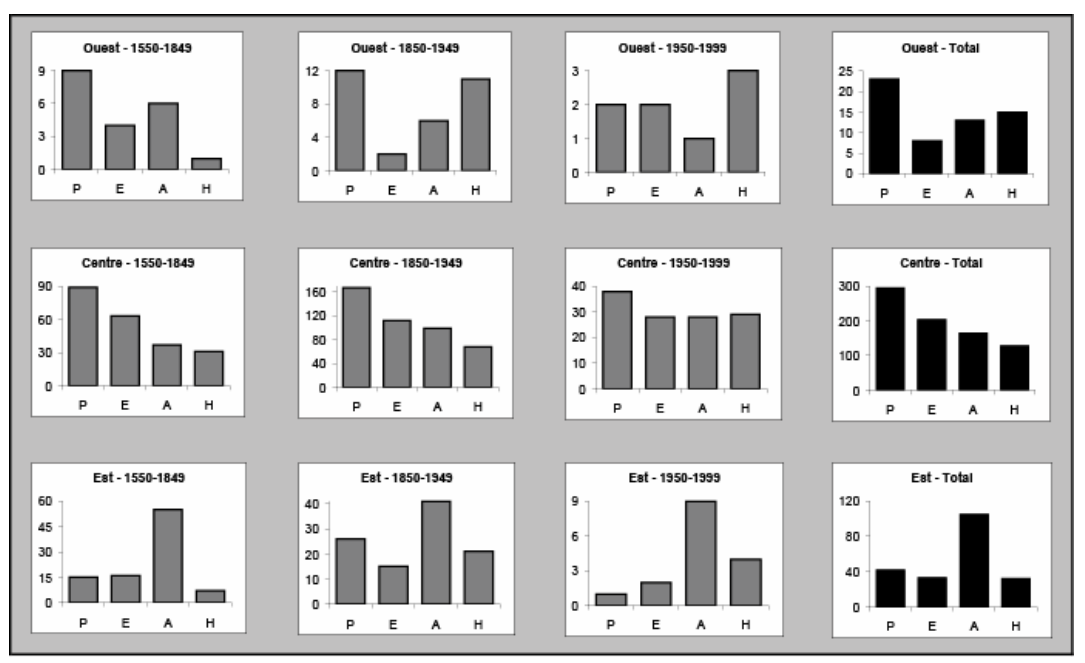

Fig. 7 - Evolution historique de la répartition saisonnière des inondations dans les Pyrénées (XVI ${ }^{\mathrm{e}}-\mathrm{XX}^{\mathrm{e}}$ siècles)

Le deuxième constat est que cette répartition a varié depuis le XVII ${ }^{\mathrm{e}}$ siècle, notoirement pour les ailes occidentale et orientale de la chaîne, moins significativement au centre (Fig. 7). La seule constante, quels que soient les secteurs considérés, est manifestement le rééquilibrage, entre le Petit Age Glaciaire et la période actuelle, vers des crues d'hiver et d'automne au détriment généralement des crues d'été ou de printemps.

L'évolution des pourcentages saisonniers du printemps et de l'été d'une part, de l'automne et de l'hiver d'autre part, adoptent d'ailleurs des évolutions identiques (Fig. 8). Cette dernière figure montre par ailleurs une distribution saisonnière des inondations chaotique, extrêmement changeante d'un demi-siècle à l'autre au cours du Petit Age Glaciaire, alors qu'elle semble beaucoup plus stable avant 1550 et, surtout, après 1850 .
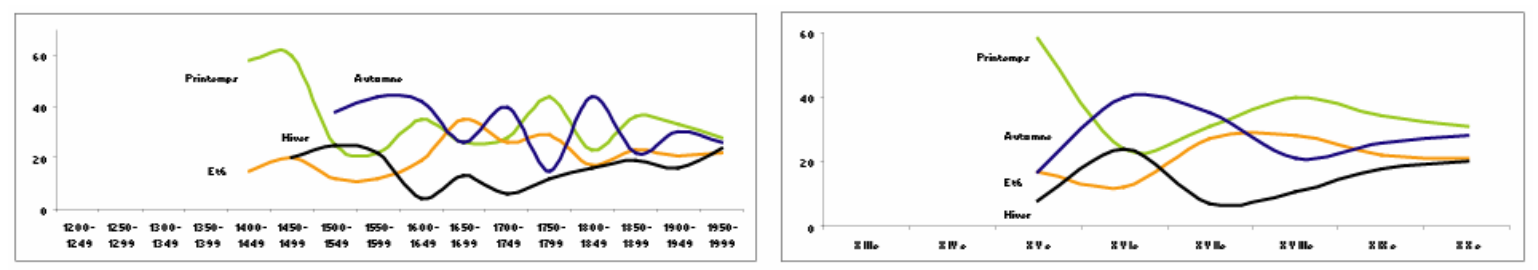

Fig. 8 - Evolution des pourcentages saisonniers des inondations par siècle et par demi-siècle dans les Pyrénées
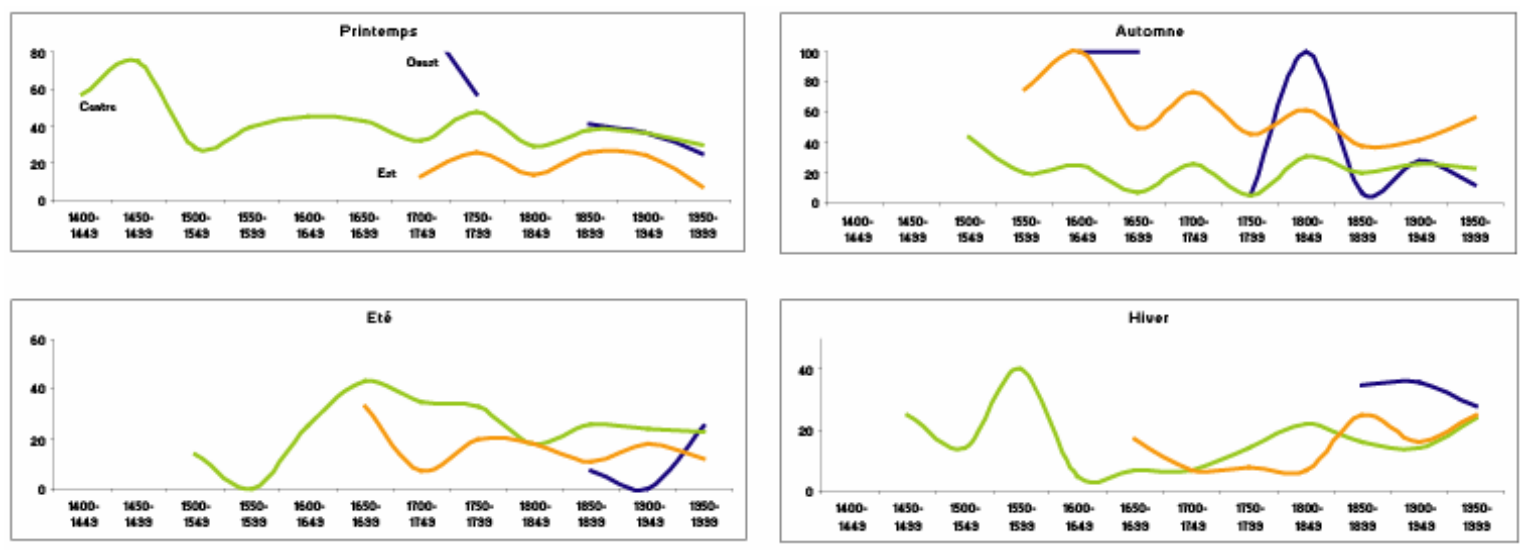

Fig. 9 - Evolution du pourcentage saisonnier des inondations par demi-siècle et par grandes régions pyrénéennes 
Enfin, sur le plan spatial, on s'aperçoit que la répartition saisonnière des phénomènes dans les aires centrale et orientale évolue de façon similaire (Fig. 9), en particulier au printemps et en automne, alors que l'aire occidentale fonctionne de façon plutôt indépendante. L'ensemble des observations faites ici semble donc aller dans le sens d'un déterminisme climatique, au moins partiel, dans la dynamique des inondations au cours du Petit Age Glaciaire.

\section{CONCLUSION}

Par rapport au questionnement initial sur l'opportunité de choisir les inondations et les crues torrentielles comme marqueurs des évolutions climatiques, il apparaît au final qu'elles peuvent être en effet de bons indicateurs. L'examen historique des inondations pyrénéennes semble bien a priori traduire la survenance du Petit Age Glaciaire, mais pas dans le sens où on aurait pu l'imaginer. Plus qu'une période homogène de crise hydrologique, c'est-à-dire caractérisée en continu par une fréquence et/ou une intensité plus grandes des phénomènes, le Petit Age Glaciaire pyrénéen se présente comme une alternance de périodes «normales » et de crises hydrologico-torrentielles. Il n'y a finalement que lors de ces crises que surviennent des événements « hors-normes » par leur intensité, leur extension spatiale, leur succession rapprochée dans le temps et/ou dans l'espace (le long de la chaîne). Cette irrégularité, qui est par ailleurs à bien des égards la signature du Petit Age Glaciaire, se retrouve également au niveau des saisons de survenance des crues : elles sont aussi très erratiques au cours de la période, avec des saisons de survenance ici aussi hors-norme lors des crises, et notamment lors de celle de la fin du XVIII ${ }^{\mathrm{e}}$ siècle. Ces interprétations de la dynamique historique des inondations et des crues torrentielles dans les Pyrénées laissent cependant plusieurs questions en suspens.

La première est liée à l'interprétation de la dynamique des inondations en termes climatiques. Quelle est la signification climatique des variations de la dynamique hydrologico-torrentielle? Quels sont précisément les facteurs et les processus climatiques entrant en jeu (températures, précipitations, circulation atmosphérique), en Europe de l'Ouest d'une manière générale, mais aussi plus particulièrement sur la chaîne pyrénéenne, à l'interface des domaines atlantique et méditerranéen ? Répondre à ces questions, c'est voir comment un changement climatique influence la dynamique des inondations, mais aussi en négatif, comment peuvent intervenir les facteurs non climatiques.

C'est alors la question de la nature des crises qui se pose : crises climatico-torrentielles ou plus largement environnementales, c'est-à-dire mettant en jeu la société, tant dans ses actions objectives (comme facteur de déclenchement) que subjectives (perception et consignation des phénomènes dans les archives) ? On ne peut en effet que constater par exemple que la crise torrentielle du XIX ${ }^{\mathrm{e}}$ siècle coïncide avec une exploitation maximale des milieux, de fait plus sensibles à la torrentialité. La réponse à ces questions passe par une meilleure connaissance de la dynamique hydrologicotorrentielle du début du Petit Age Glaciaire, et plus globalement pour la période, par le recoupement des tendances hydrologiques avec d'autres indicateurs, notamment climatiques. On pense en particulier à l'évolution de la NAO (North Atlantic Oscillation) et de la circulation atmosphérique sur l'Europe de l'Ouest. 


\section{Bibliographie}

ALEXANDRE (P.), Le climat en Europe au Moyen-Age. Contribution à l'histoire des variations climatiques de 1000 à 1425, d'après les sources narratives de l'Europe occidentale, Paris, EHESS, 1987, 827 p.

ALLARD (P.), Eléments pour une problématique de l'histoire du risque. Du risque accepté au risque maîtrisé. Représentations et gestion du risque d'inondation en Camargue, XVIII ${ }^{e}-X I X^{e}$ siècles, HDR, Université d'Aix-Marseille II, 2000, 200 p.

ANTOINE (J.-M.), La catastrophe oubliée. Les avatars de l'inondation, du risque et de l'aménagement dans la vallée de l'Ariège (Pyrénées françaises, fin $X V I I^{e}-X X^{e}$ siècles), Thèse de doctorat de Géographie, Université de Toulouse-Le Mirail, 1992, 495 p.

ANTOINE (J.-M.), Catastrophes torrentielles et géographicité des sources historiques. Le cas de la Baronnie de ChâteauVerdun (Pyrénées ariégeoises) au XVIII ${ }^{\mathrm{e}}$ siècle, Sources, Travaux historiques, 33, 1993, pp. 51-63.

ANTOINE (J.-M.), Histoire et temporalités des catastrophes et des risques « naturels». Eléments de prospective dans le contexte du changement climatique, Actes du colloque "Risques environnementaux et changement climatique. Quelles réponses sociales? », Grenoble, MSH Alpes, novembre 2007, a paraître.

ANTOINE (J.-M.), DESAILLY (B.), METAILIE (J.P.), La chronologie des crues et phénomènes torrentiels dans les Pyrénées $\left(\mathrm{XVIII}^{\mathrm{e}}-\mathrm{XX}^{\mathrm{e}}\right.$ siècles). Premiers résultats et problèmes d'interprétation, in: Risques et aménagement dans les Pyrénées, Les Cahiers de l'ISARD, $n^{\circ} 4$, Toulouse, CNRS-Groupement de Recherche Information Scientifique sur l'Aménagement Régional et le Développement, 1993, p. 31-58.

ANTOINE (J.-M.), DESAILLY (B.), METAILIE (J.P.), Les grands aïgats du $\mathrm{XVIII}^{\mathrm{e}}$ siècle dans les Pyrénées, in: Les catastrophes naturelles dans l'Europe médiévale et moderne, Actes des $\mathrm{XV}^{\text {èmes }}$ Journées Internationales d'Histoire de Flaran, Toulouse, Presses Universitaires du Mirail, 1995, p. 243-260.

ANTOINE (J.-M.), DESAILLY (B.), Etude de faisabilité de bases de données historiques sur les inondations - Base nationale et bases départementales, Rapport, 41 p., base de données informatisée Accès ${ }^{\mathrm{TM}}$ (Microsoft) sur le département du Gers, GEODE UMR-5602 CNRS, Ministère de l'Environnement et de l'Aménagement du Territoire, CERTU Lyon, 2000.

ANTOINE (J.-M.), DESAILLY (B.), HURAND (A.), LANUSSE (M.), Méthodologie de recherche historique pour une cartographie des risques naturels dans les Pyrénées Application à une zone test comprenant les communes de la zone montagne du département de la Haute-Garonne, Rapport, 53 p., base de données informatisée sur la zone test, DIREN MidiPyrénées, Délégation RTM «Pyrénées », GEODE UMR-5602 CNRS, Université de Pau et des Pays de l'Adour, 2001.

ARNAUD-FASSETTA (G.), PROVENSAL (M.), High frequency variations of water flux and sediment discharge during the Little Ice Age (1586-1725 AD) in the Rhône Delta (Mediterranean France). Relationship to the catchment basin, Hydrobiologia, 410, 1999, p. 241-250.

BARRIENDOS VALLVÉ (M.), Les inundacions al Pla de Barcelona (segles XIV-XIX). Aspectes paleoclimatics del fenomen, Actes du IIIe Congrès d'Histoire de Barcelone, Ajuntament de Barcelona, 1993, p. 275-280.

BARRIENDOS VALLVÉ (M.), MARTIN-VIDE (J.), Secular climatic oscillations as indicated by catastrophic floods in the spanish mediterranean coastal area (14th-19th centuries), Climatic Change, 38, 1998, pp. 473-491.

BECK (C.), DELORT (R.) (dir.), Pour une histoire de l'environnement et des phénomènes naturels, Paris, Éditions du C.N.R.S., 1993, 272 p.

BENITO (G.), MACHADO (M.J.), PEREZ-GONZALEZ (A.), Climate change and flood sensitivity in Spain, in BRANSON (J.), BROWN (A.G.) \& GREGORY (K.J.), éd., Global continental changes: the context of paleohydrology, London, Geological Society Special Publication $N^{\circ} 115,1996$, p. 85-98.

BENNASSAR (B.) éd., Les catastrophes naturelles dans l'Europe médiévale et moderne, Toulouse, PUM, 1996, 275 p.

BERLIOZ (J.), L'effondrement du Mont Granier en Savoie (fin 1248), Le monde alpin et rhodanien, 1987, p. 7-68.

BERLIOZ (J.), Les récits exemplaires, sources imprévues de l'histoire des catastrophes naturelles au Moyen-Age, Sources, Travaux historiques, $\mathrm{n}^{\circ} 33,1993, \mathrm{p} .7-24$

BRAVARD (J.-P.), La crise environnementale: entre faits objectifs et construits sociaux, in : BECK (C.), LUGINBÜHL (Y.), MUXART (T.), éd., Temps et espaces des crises de l'environnement, Versailles, Editions Quae, 2006, p. 149-156.

BRAZDIL (R.), The history of floods on the rivers Elbe and Vlatava in Bohemia, Erfurter Geographische Studien, 7, 1998, p. 93-108.

BRAZDIL (R.), GLASER (R.), PFISTER (C.), DOBROVOLNY (P.), ANTOINE (J.-M.), BARRIENDOS (M.), CAMUFFO (D.), DEUTSCH (M.), ENZI (S.), GUIDOBONI (E.), KOTIZA (O.), RODRIGO (F.S.), Floods events of selected european rivers in the sixteenth Century, Climatic Change, volume 43, $\mathrm{n}^{\circ} 1,1999$, p.239285 .

BRIFFAUD (S.), Le rôle des catastrophes naturelles : le cas des Pyrénées centrales, in : CORVOL (A.), éd., La nature en révolution, 1750-1800, Paris, L'Harmattan, 1993, p. 134-144.

BRIFFAUD (S.), Naissance d'un paysage : la montagne pyrénéenne à la croisée des regards (XVI ${ }^{e}-X I X^{e}$ siècle). Toulouse, AGM, CNRS, 1994, 529 p.

CEEUR (D.), LANG (M.), L'information historique des inondations : l'histoire ne donne-t-elle que des leçons ?, La Houille Blanche, 2, 2000, p. 79-84.

CEEUR (D.), La plaine de Grenoble face aux inondations. Genèse d'une politique publique du XVII au XX siècle, Versailles, Editions Quae, 2008, 328 p. (édition de la thèse soutenue en 2003 à l'université de Grenoble).

CORVOL (A.) éd., Les sources de l'histoire de l'environnement, le XIX $X^{e}$ siècle, Paris, L'Harmattan, 1999, 502 p.

CORVOL (A.) éd., Les sources de l'histoire de l'environnement, le $X X^{e}$ siècle, Paris, L'Harmattan, 2003, 750 p.

CORVOL (A.) et ROCHEFORT (I.), éd., Nature, environnement et paysage. L'héritage du XVIII é siècle. Guide de recherches archivistiques et bibliographiques, Paris, L'Harmattan, 1995, 295 p.

DELORT (R.), WALTER (F.), Histoire de l'environnement européen, Paris, PUF, 2001, $354 \mathrm{p}$.

DELUMEAU (J.) et LEQUIN (Y.) éd., Les malheurs du temps. Histoire des fléaux et des calamités en France, Paris, Larousse, 1991, 519 p.

DESAILLY (B.), Crues et inondations en Roussillon. Le risque et l'aménagement, fin $d u X V I I^{e}-$ milieu $d u X X^{e}$ siècles, Thèse de 
doctorat de Géographie, Université de Paris X-Nanterre, 1990, $352 \mathrm{p}$

DESPLAT (Ch.), Séismes dans les Pyrénées occidentales. Contribution à l'histoire des phénomènes naturels, Revue Géographique des Pyrénées et du Sud-Ouest, 59, 1, 1988, p. 99110

DESPLAT (Ch.), Pour une histoire des risques naturels dans les Pyrénées occidentales françaises sous l'Ancien Régime, Revue de Pau et du Béarn, 21, 1994, p. 101-156.

FAGAN (B. M.), The Little Ice Age : how climate made history, 1300-1850, New-York, Basic Books, 2000, 272 p

FAVIER (R.) éd., Les pouvoirs publics face aux risques naturels dans l'histoire, Grenoble, CNRS-MSH-Alpes, 2002, 444 p.

FAVIER (R.) et GRANET-ABISSET (A.-M.) éd., Récits et représentations des catastrophes depuis l'Antiquité, Grenoble, CNRS-MSH-Alpes, 2005, $408 \mathrm{p}$

GALABERT (F.), Dépouillement des archives de la ville de Toulouse à propos des inondations de la Garonne, dossier manuscrit, archives de la ville de Toulouse, 1918, côte B 88

GARNIER (E.), Risques d'inondations et aménagements dans l'orne $\mathrm{XVII}^{\mathrm{e}}-\mathrm{XIX}^{\mathrm{e}}$ siècles, Société historique et archéologique de l'Orne, 2, 125, 2006a, p. 39-60.

GARNIER (E.), Programme d'Histoire du climat « ophélie », Annales de Normandie, 1, 56, 2006b, p. 93-94.

GLASER (R.), STANGL (H.), Historical floods in the Dutch Rhine Delta, Natural Hazards and Earth System Sciences, 3, 2003 , p. $1-9$

GLASER (R.), STANGL (H.), Climate and floods in Central Europe since AD 1000 : data, methods, results and consequences, Surveys in Geophysics, 25, 2004, p. 485-510.

GOTTSCHALK (M.K.E.), Stormvloeden in rivieroverstroomingen in Nederland, Assen, Amsterdam, Van Gorcum, 1971-1977, 3 tomes.

GRANET-ABISSET (A.-M.) et FAVIER (R.) éd., Histoire et mémoire des risques naturels, Grenoble, CNRS-MSH-Alpes, 2001, $282 \mathrm{p}$.

HOUGHTON (J.T.), DING (Y.), GRIGGS (D.J.), NOGUER (M.), VAN DER LINDEN (P.J.), XIAOSU (D.), Bilan 2001 des changements climatiques : Les éléments scientifiques, Contribution du groupe de travail I au $3^{\text {ème }}$ rapport du GIEC, OMM/PNUE, 2001, 97 p.

LAMB (H. H.), Climate - Present, Past and Future, volume 2, Climatic history and the future, Londres, Methuen, 1977, 837 p.

LAMB (H.H.), Historic Storms Of The North Sea, British Isles And Northwest Europe, New-York, Cambridge University Press, 1991, $204 \mathrm{p}$.

LAMB (H. H.), Climate, history and the modern world, NewYork, Routledge, 1995, 433 p.

LE ROY LADURIE (E.), Histoire du climat depuis l'An Mil, Paris, Flammarion, 1967, 374 p.

MASSARD-GUILBAUD (G.), De la « part du milieu » à l'histoire de l'environnement, Le Mouvement Social, 200, 2002, p. 64-72.

MESCHINET DE RICHEMOND (N.), Les inondations catastrophiques sur la bordure montagneuse du Roussillon : dégâts et sinistres. Thèse de doctorat de Géographie, Université de Paris X-Nanterre, 1997, 355 p.

METAILIE (J.-P.), The degradation of the Pyrenees in the Nineteenth century - an erosion crisis ?, in : GARDINER (V.), éd., Proceedings of the First International Conference on Geomorphology, Manchester, september 1985, Chichester, J. Wiley, 1987, Part. II, p. 533-544.

METAILIE (J.-P.) éd., Le torrent et le fleuve. Risques, Catastrophes et aménagement dans les Pyrénées et sur leur piémont, Rapport final d'ATP PIREN-CNRS, GEODE UMR 5602CNRS, Université de Toulouse-Le Mirail, 1991, 315 p.

METAILIE (J.-P.), La dégradation des montagnes au XIX ${ }^{\mathrm{e}}$ siècle dans les Pyrénées, in : BECK (C.), LUGINBÜHL (Y.), MUXART (T.), éd., Temps et espaces des crises de l'environnement, Versailles, Editions Quae, 2006, p. 191-210.

MIRAMONT (C.), GUILBERT (X.), Variations historiques de la fréquence des crues et évolution de la morphogenèse fluviale en moyenne Durance (France du Sud-Est), Géomorphologie : relief, processus, environnement, 4, 1997, p. 325-338.

PACHAURI (R.K.) et REISINGER (A.), éd., Bilan 2007 des changements climatiques. Contribution des Groupes de travail I, II et III au quatrième Rapport d'évaluation du Groupe d'experts intergouvernemental sur l'évolution $d u$ climat, Genève, GIEC, 2007, 103 p.

PAVESE (M.P.), BANZON (V.), COLACINO (M.), GREGORI (G.P.), PASQUA (M.), Three historical data series on floods and anomalous climatic events in Italy, in : BRADLEY (R.S.), JONES (P.D.), éd., Climate since AD 1500, London, Routledge, 1995, p. 155-170.

PICON (B.), ALLARD (P.), CLAEYS-MEKDALE (C.), KILLIAN (S.), Gestion du risque inondation et changement social dans le delta du Rhône. Les catastrophes de 1856 et 1993-1994, Versailles, Editions Quae, 2006, 122 p.

PFISTER (Ch.), Klimageschichte der Schweiz 1525-1860, Das Klima der Schweiz von 1525-1860 und seine Bedeutung in der Geschichte von Bevölkerung und Landwirtschaft, Bern et Stuttgart, 1988, 2 vol.

PICHARD (G.), Les crues sur le bas-Rhône de 1500 à nos jours. Pour une histoire hydro-climatique, Méditerranée, 3-4, 1995, p. 105-116.

PICHARD (G.), Espaces et nature en Provence - L'environnement rural 1540-1789, Aix-Marseille, Université de Provence, 1999, 3 vol..

PICHARD (G.), Torrents et société à Vergons au temps du « Petit Age Glaciaire », Méditerranée, 1-2, 2004, p. 119-126.

ROHR (CH.), The Danube Floods and Their Human Response and Perception (14th to 17th C), History of Meteorology, 2, 2005, p. 71-86.

SMEPAG, Monographie des crues de la Garonne (du Pont du Roy au Bec d'Ambès), 1989, 2 tomes.

WALTER (F.), Les Suisses et l'environnement. Une histoire du rapport à la nature du XVIII siècle à nos jours. Genève, Editions Zoé, 1990, 296 p

WALTER F., Catastrophes. Une histoire culturelle, $X V I^{e}-X X I^{e}$ siècle, Paris, Seuil, 2008, 380 p.

WALTER (F.), FANTINI (B.), DELVAUX (P.), éd., Les cultures $d u$ risque $\left(16^{e}-21^{e}\right.$ siècle), Genève, Presses d'Histoire Suisses, 2006. 